Vol. 54 (1996) [509-516]

\title{
HIGHER-ORDER OPTIMALITY CONDITIONS FOR A MINIMAX
}

\author{
Do Van LuU and W. Oettli
}

\begin{abstract}
Higher-order necessary and sufficient optimality conditions for a nonsmooth minimax problem with infinitely many constraints of inequality type are established under suitable basic assumptions and regularity conditions.
\end{abstract}

\section{INTRODUCTION}

Let $C$ be a nonempty subset of a normed space $X$, and let $Q$ and $B$ be nonempty sets. Let $f_{\alpha}(\alpha \in Q)$ and $g_{\beta}(\beta \in B)$ be real-valued functions on $X$. We consider the following minimax problem:

$$
\min \{F(x) \mid x \in C, G(x) \leqslant 0\}
$$

where $F(x):=\sup _{\alpha \in Q} f_{\alpha}(x)$ and $G(x):=\sup _{\beta \in B} g_{\beta}(x)$.

Our aim here is to develop higher-order optimality conditions for (P) by using suitable approximations to the functions involved. Thus our results will be formulated in terms of approximating functions $\phi_{\alpha}^{(k)}($.$) and \psi_{\beta}^{(k)}($.$) . These may be thought of as$ substitutes for the $k$-th order directional derivatives of $f_{\alpha}$ and $g_{\beta}$, but are considerably more general. For instance, $\phi_{\alpha}^{(k)}($.$) and \psi_{\beta}^{(k)}($.$) do not need to be positively$ homogeneous of degree $k$. The lack of homogeneity forces us to use a particular kind of regularity condition (namely condition 2.3 below). Our approach extends the technique we have used in [6] to derive first-order conditions. Some optimality conditions from [8] are included as special cases in our results.

\section{HIGHER-ORDER NECESSARY OPTIMALITY CONDITIONS}

In the following we fix a reference point $x_{0} \in C$ which is feasible for problem (P). We assume that $F\left(x_{0}\right)$ is finite. Let

$$
Q_{0}:=\left\{\alpha \in Q \mid f_{\alpha}\left(x_{0}\right)=F\left(x_{0}\right)\right\}, \quad B_{0}:=\left\{\beta \in B \mid g_{\beta}\left(x_{0}\right)=G\left(x_{0}\right)\right\}
$$

Received 9th January, 1996

The help of Dirk Schläger in editing the final version of this paper is gratefully acknowledged.

Copyright Clearance Centre, Inc. Serial-fee code: 0004-9729/96 \$A2.00+0.00. 
We assume that $Q, B$ are compact topological spaces, and that the mappings $\alpha \mapsto$ $f_{\alpha}\left(x_{0}\right)$ and $\beta \mapsto g_{\beta}\left(x_{0}\right)$ are upper semicontinuous. Then $Q_{0}$ and $B_{0}$ are nonempty and compact. We recall $\left[2\right.$, p.55] that the contingent cone to $C$ at $x_{0}$ is the set

$$
K_{C}\left(x_{0}\right):=\left\{d \in X \mid \exists\left\{d_{n}\right\} \subseteq X,\left\{t_{n}\right\} \subseteq \mathbb{R}: d_{n} \rightarrow d, t_{n} \downarrow 0, x_{0}+t_{n} d_{n} \in C\right\}
$$

To derive necessary optimality conditions for $(P)$, we introduce functions which play the roles of higher-order generalised directional derivatives of $f_{\alpha}$ and $g_{\beta}$. So, for each $\alpha \in Q, \beta \in B$, let $\varphi_{\alpha}^{(i)}, i \in I:=\{1, \ldots, k\}$, and $\psi_{\beta}^{(j)}, j \in J:=\{1, \ldots, p\}$, be real-valued functions on $X$ satisfying the following:

\section{AsSUMPTION 2.1 .}

(a) $\varphi_{a}^{(i)}(0)=0, \psi_{\beta}^{(j)}(0)=0$ for all $\alpha \in Q, \beta \in B, i \in I, j \in J$.

(b) The mappings $\alpha \mapsto \varphi_{\alpha}^{(i)}(d)$ and $\beta \mapsto \psi_{\beta}^{(j)}(d)$ are continuous for all $d \in$ $K_{C}\left(x_{0}\right), i \in I, j \in J$.

(c) If $d_{n} \rightarrow d$ as $n \rightarrow \infty$, then, for each $i \in I$,

$$
\liminf _{n \rightarrow \infty}\left[\varphi_{\alpha}^{(i)}\left(d_{n}\right)-\varphi_{\alpha}^{(i)}(d)\right] \leqslant 0 \quad \text { uniformly in } \alpha,
$$

and, for each $j \in J$,

$$
\liminf _{n \rightarrow \infty}\left[\psi_{\beta}^{(j)}\left(d_{n}\right)-\psi_{\beta}^{(j)}(d)\right] \leqslant 0 \quad \text { uniformly in } \beta \text {. }
$$

(d) The mapping $d \mapsto \max _{\alpha \in Q_{0}} \varphi_{\alpha}^{(k)}(d)$ is upper semicontinuous.

Let us introduce relations between $f_{\alpha}$ and $\varphi_{\alpha}^{(k)}, g_{\beta}$ and $\psi_{\beta}^{(p)}$.

Basic Assumption 2.2. For all $d \in K_{C}\left(x_{0}\right)$ and sequences $d_{n} \rightarrow d$, $t_{n} \downarrow 0$ satisfying $x_{0}+t_{n} d_{n} \in C$,

$$
\varphi_{\alpha}^{(k)}(d) \geqslant \liminf _{n \rightarrow \infty} \frac{1}{t_{n}^{k}}\left[f_{\alpha}\left(x_{0}+t_{n} d_{n}\right)-f_{\alpha}\left(x_{0}\right)-\sum_{i=1}^{k-1} t_{n}^{i} \varphi_{\alpha}^{(i)}\left(d_{n}\right)\right] \quad \text { uniformly in } \alpha,
$$

and

$$
\psi_{\beta}^{(p)}(d) \geqslant \liminf _{n \rightarrow \infty} \frac{1}{t_{n}^{p}}\left[g_{\beta}\left(x_{0}+t_{n} d_{n}\right)-g_{\beta}\left(x_{0}\right)-\sum_{j=1}^{p-1} t_{n}^{j} \psi_{\beta}^{(j)}\left(d_{n}\right)\right] \quad \text { uniformly in } \beta
$$

To proceed further let us introduce the sets

$$
\begin{aligned}
& M\left(x_{0}\right):=\left\{d \in K_{C}\left(x_{0}\right) \mid \max _{\alpha \in Q_{0}} \varphi_{\alpha}^{(i)}(d) \leqslant 0 \forall i \in I \backslash\{k\}, \max _{\beta \in B_{0}} \psi_{\beta}^{(j)}(d) \leqslant 0 \forall j \in J\right\}, \\
& \widetilde{M}\left(x_{0}\right):=\left\{d \in K_{C}\left(x_{0}\right) \mid \max _{\alpha \in Q_{0}} \varphi_{\alpha}^{(i)}(d)<0 \forall i \in I \backslash\{k\}, \max _{\beta \in B_{0}} \psi_{\beta}^{(j)}(d)<0 \forall j \in J\right\} .
\end{aligned}
$$


Moreover, for $V \subseteq Q$ and $W \subseteq B$ let us define

$$
\mathcal{C}(V, W):=\left\{d \in K_{C}\left(x_{0}\right) \mid \varphi_{\alpha}^{(i)}(d)<0 \forall \alpha \in V, i \in I ; \psi_{\beta}^{(j)}(d)<0 \forall \beta \in W, j \in J\right\} .
$$

Let us introduce a regularity condition of the type used in [3].

\section{Regularity Condition 2.3.}

(i) For any closed sets $V$ and $W$ satisfying $Q_{0} \subseteq V \subseteq Q$ and $B_{0} \subseteq W \subseteq B$ it holds that $\mathcal{C}(V, W) \neq \emptyset$ implies $0 \in \operatorname{cl} \mathcal{C}(V, W)$.

(ii) $M\left(x_{0}\right) \subseteq \operatorname{cl} \widetilde{M}\left(x_{0}\right)$.

Note that 2.3(i) holds, if the functions $\varphi_{\alpha}^{(i)}, \psi_{\beta}^{(j)}$ are positively homogeneous.

We are now in a position to formulate a general necessary optimality condition of order $k$ for $(\mathrm{P})$, which is the main result of the paper.

Theorem 2.4. Let $x_{0}$ be a local minimiser for (P). Assume that assumption 2.1 , the basic assumption 2.2 , and the regularity condition 2.3 hold. Then

$$
\max _{\alpha \in Q_{0}} \varphi_{\alpha}^{(k)}(d) \geqslant 0 \quad \forall d \in M\left(x_{0}\right)
$$

Proof: Suppose that (1) is not true. By 2.3.(ii), there exists $\bar{d} \in \operatorname{cl} \widetilde{M}\left(x_{0}\right)$ such that

$$
\max _{\alpha \in Q_{0}} \varphi_{\alpha}^{(k)}(d)<0 .
$$

By 2.1.(d), $d \mapsto \max _{\alpha \in Q_{0}} \varphi_{\alpha}^{(k)}(d)$ is upper semicontinuous. So we can assume that $\bar{d} \in$ $\widetilde{M}\left(x_{0}\right)$, that is, $\bar{d} \in K_{C}\left(x_{0}\right)$ and

$$
\max _{\alpha \in Q_{0}} \varphi_{\alpha}^{(i)}(d)<0 \quad \forall i \in I, \quad \max _{\beta \in B_{0}} \psi_{\beta}^{(j)}(d)<0 \quad \forall j \in J
$$

We choose $\delta>0$ such that $\varphi_{\alpha}^{(i)}(d) \leqslant-2 \delta$ for all $\alpha \in Q_{0}, i \in I$, and $\psi_{\beta}^{(j)}(d) \leqslant-2 \delta$ for all $\beta \in B_{0}, j \in J$. We define

$$
U_{1}:=\left\{\alpha \in Q \mid \varphi_{\alpha}^{(i)}(d)<-\delta \forall i \in I\right\}, \quad U_{2}:=\left\{\beta \in B \mid \psi_{\beta}^{(j)}(d)<-\delta \forall j \in J\right\} .
$$

Then $Q_{0} \subseteq U_{1}, B_{0} \subseteq U_{2}$. By 2.1.(b), $U_{1}$ and $U_{2}$ are open, and

$$
\varphi_{\alpha}^{(i)}(d) \leqslant-\delta \quad \forall \alpha \in \operatorname{cl} U_{1}, i \in I, \quad \psi_{\beta}^{(j)}(d) \leqslant-\delta \quad \forall \beta \in \operatorname{cl} U_{2}, j \in J .
$$

So $\bar{d} \in \mathcal{C}\left(\operatorname{cl} U_{1}, \operatorname{cl} U_{2}\right)$ and, by 2.3.(i), $0 \in \operatorname{cl} \mathcal{C}\left(\operatorname{cl} U_{1}, \operatorname{cl} U_{2}\right)$. Hence there exists a sequence $\left\{h_{n}\right\} \subseteq \mathcal{C}\left(\operatorname{cl} U_{1}, \mathrm{cl} U_{2}\right)$ converging to 0 . 
We assert that there exist $d \in K_{C}\left(x_{0}\right)$ and $\varepsilon>0$ satisfying

$$
\begin{array}{ll}
\varphi_{\alpha}^{(i)}(d)+\varepsilon \leqslant F\left(x_{0}\right)-f_{\alpha}\left(x_{0}\right) & \forall i \in I, \\
\psi_{\beta}^{(j)}(d)+\varepsilon \leqslant G\left(x_{0}\right)-g_{\beta}\left(x_{0}\right) & \forall j \in J
\end{array}
$$

for every $\alpha \in Q, \beta \in B$. To prove this, observe that $f_{\alpha}\left(x_{0}\right)<F\left(x_{0}\right)$ for all $\alpha \in Q \backslash U_{1}$ and $g_{\beta}\left(x_{0}\right)<G\left(x_{0}\right)$ for all $\beta \in B \backslash U_{2}$. Since $Q \backslash U_{1}, B \backslash U_{2}$ are compact and $\alpha \mapsto f_{\alpha}\left(x_{0}\right), \beta \mapsto g_{\beta}\left(x_{0}\right)$ are upper semicontinuous, there exists $\varepsilon_{1}>0$ such that

$$
f_{\alpha}\left(x_{0}\right) \leqslant F\left(x_{0}\right)-2 \varepsilon_{1} \quad \forall \alpha \in Q \backslash U_{1}, \quad g_{\beta}\left(x_{0}\right) \leqslant G\left(x_{0}\right)-2 \varepsilon_{1} \quad \forall \beta \in B \backslash U_{2} .
$$

Since $h_{n} \rightarrow 0$, by 2.1.(a) and (c) there exists $m \in \mathbb{N}$ with

$$
\varphi_{\alpha}^{(i)}\left(h_{m}\right) \leqslant \varepsilon_{1} \quad \forall \alpha \in Q, i \in I, \quad \psi_{\beta}^{(j)}\left(h_{m}\right) \leqslant \varepsilon_{1} \quad \forall \beta \in B, j \in J
$$

Let $d:=h_{m}$. Then (2) holds for all $\alpha \in Q \backslash U_{1}, \beta \in B \backslash U_{2}$, and every $\varepsilon \leqslant \varepsilon_{1}$. On the other hand, $d \in \mathcal{C}\left(\mathrm{cl} U_{1}, \mathrm{cl} U_{2}\right)$. Hence by 2.1.(b) and the compactness of $\operatorname{cl} U_{1}, \operatorname{cl} U_{2}$ there exists $\varepsilon_{2}>0$ such that

$$
\varphi_{\alpha}^{(i)}(d)+\varepsilon_{2} \leqslant 0 \quad \forall \alpha \in \operatorname{cl} U_{1}, i \in I, \quad \psi_{\beta}^{(j)}(d)+\varepsilon_{2} \leqslant 0 \quad \forall \beta \in \operatorname{cl} U_{2}, j \in J .
$$

This implies (2) for all $\alpha \in \operatorname{cl} U_{1}, \beta \in \operatorname{cl} U_{2}$, and every $\varepsilon \leqslant \varepsilon_{2}$.

Now we use (2) to find a sequence $\left\{x_{n}\right\}$ of feasible points for problem (P) converging to $x_{0}$ such that $F\left(x_{n}\right)<F\left(x_{0}\right)$ for all $n$, which contradicts the hypothesis that $x_{0}$ is a local minimiser.

Since $d \in K_{C}\left(x_{0}\right)$, there exist sequences $\left\{d_{n}\right\} \subseteq X,\left\{t_{n}\right\} \subseteq \mathbb{R}$ such that $d_{n} \rightarrow d$, $t_{n} \downarrow 0$, and $x_{n}:=x_{0}+t_{n} d_{n} \in C$ for all $n$. Replacing these by appropriate subsequences we can assume that, for every $n$,

$$
\begin{array}{ll}
\frac{1}{t_{n}^{k}}\left[f_{\alpha}\left(x_{n}\right)-f_{\alpha}\left(x_{0}\right)-\sum_{i=1}^{k-1} t_{n}^{i} \varphi_{\alpha}^{(i)}\left(d_{n}\right)\right] \leqslant \varphi_{\alpha}^{(k)}(d)+\frac{\varepsilon}{2} & \forall \alpha \in Q, \\
\frac{1}{t_{n}^{p}}\left[g_{\beta}\left(x_{n}\right)-g_{\beta}\left(x_{0}\right)-\sum_{j=1}^{p-1} t_{n}^{j} \psi_{\beta}^{(j)}\left(d_{n}\right)\right] \leqslant \psi_{\beta}^{(p)}(d)+\frac{\varepsilon}{2} & \forall \beta \in B
\end{array}
$$

by 2.2 ,

$$
\begin{array}{ll}
\varphi_{\alpha}^{(i)}\left(d_{n}\right) \leqslant \varphi_{\alpha}^{(i)}(d)+\varepsilon & \forall \alpha \in Q, i \in I, \\
\psi_{\beta}^{(j)}\left(d_{n}\right) \leqslant \psi_{\beta}^{(j)}(d)+\varepsilon & \forall \beta \in B, j \in J
\end{array}
$$


by 2.1.(c), and $\sum_{i=1}^{k} t_{n}^{i} \leqslant 1, \sum_{j=1}^{p} t_{n}^{j} \leqslant 1$. Then, for all $\alpha \in Q$,

$$
\begin{aligned}
f_{\alpha}\left(x_{n}\right) & \leqslant t_{n}^{k}\left(\varphi_{\alpha}^{(k)}(d)+\frac{\varepsilon}{2}\right)+\sum_{i=1}^{k-1} t_{n}^{i} \varphi_{\alpha}^{(i)}\left(d_{n}\right)+f_{\alpha}\left(x_{0}\right) \\
& \leqslant t_{n}^{k}\left(\varphi_{\alpha}^{(k)}(d)+\varepsilon\right)+\sum_{i=1}^{k-1} t_{n}^{i}\left(\varphi_{\alpha}^{(i)}(d)+\varepsilon\right)+f_{\alpha}\left(x_{0}\right)-t_{n}^{k} \frac{\varepsilon}{2} \\
& \leqslant \sum_{i=1}^{k} t_{n}^{i}\left(F\left(x_{0}\right)-f_{\alpha}\left(x_{0}\right)\right)+f_{\alpha}\left(x_{0}\right)-t_{n}^{k} \frac{\varepsilon}{2} \\
& \leqslant F\left(x_{0}\right)-t_{n}^{k} \frac{\varepsilon}{2}
\end{aligned}
$$

and similarly $g_{\beta}\left(x_{n}\right) \leqslant G\left(x_{0}\right)-t_{n}^{k} \varepsilon / 2$ for all $\beta \in B$. Hence $F\left(x_{n}\right)<F\left(x_{0}\right)$ and $G\left(x_{n}\right)<G\left(x_{0}\right) \leqslant 0$.

In what follows, we give an application of Theorem 2.4.

EXAMPLE 2.5: Recall that $\bar{f}_{\alpha}^{(k)}\left(x_{0} ; d\right)$, the upper Dini directional derivative of order $k$ of $f_{\alpha}$ at $x_{0}$ in the direction $d$, is defined recursively as follows (see for example, $[4,5,8])$ :

$$
\bar{f}_{\alpha}^{(k)}\left(x_{0} ; d\right):=k ! \limsup _{h \rightarrow d, t \downarrow 0} \frac{1}{t^{k}}\left[f_{\alpha}\left(x_{0}+t h\right)-f_{\alpha}\left(x_{0}\right)-\sum_{i=1}^{k-1} \frac{t^{i} \bar{f}_{\alpha}^{(i)}\left(x_{0} ; h\right)}{i !}\right],
$$

provided that each $\vec{f}_{\alpha}^{(i)}$ is real-valued.

Note that the mapping $d \mapsto \bar{f}_{\alpha}^{(k)}\left(x_{0} ; d\right)$ is upper semicontinuous. By applying Theorem 2.4 to the upper Dini directional derivatives we obtain:

Corollary 2.6. Let $x_{0}$ be a local minimiser for (P). Assume that for

$$
\varphi_{\alpha}^{(i)}(d):=\frac{1}{i !} \bar{f}_{\alpha}^{(i)}\left(x_{0} ; d\right) \quad \forall i \in I, \quad \psi_{\beta}^{(j)}(d):=\frac{1}{j !} \bar{g}_{\beta}^{(j)}\left(x_{0} ; d\right) \quad \forall j \in J,
$$

assumptions 2.1.(b)-(d) and 2.3.(ii) hold. Suppose, in addition, that the limits in the definitions of $\bar{f}_{\alpha}$ and $\bar{g}_{\beta}$ at $x_{0}$ are uniformly in $\alpha$ and $\beta$, respectively. Then

$$
\max _{\alpha \in Q_{0}} \widehat{f}_{\alpha}^{(k)}\left(x_{0} ; d\right) \geqslant 0
$$

holds for every $d \in K_{C}\left(x_{0}\right)$ with

$$
\max _{\alpha \in Q_{0}} \bar{f}_{\alpha}^{(i)}\left(x_{0} ; d\right) \leqslant 0 \quad \forall i \in I \backslash\{k\}, \max _{\beta \in B_{0}} \bar{g}_{\beta}^{(j)}\left(x_{0} ; d\right) \leqslant 0 \quad \forall j \in J .
$$


PROOF: It is easy to see that assumption 2.1.(a) and the basic assumption 2.2 are satisfied. Moreover, 2.3.(i) holds since $\bar{f}_{\alpha}^{(i)}, \bar{g}_{\beta}^{(j)}$ are positively homogeneous. So the conclusion follows from Theorem 2.4.

EXAMPLE 2.7: If $f_{\alpha}$ is $(k-1)$ times Fréchet differentiable on $X(k>1)$ and the Fréchet derivative of order $k$ of $f_{\alpha}$ at $x_{0}, f_{\alpha}^{(k)}\left(x_{0}\right)$, exists, then (see for example, [7])

$$
f_{\alpha}^{(k)}\left(x_{0}\right) d^{k}=\bar{f}_{\alpha}^{(k)}\left(x_{0} ; d\right) \quad \forall d \in X,
$$

where $d^{k}:=(d, \ldots, d) \in X^{k}$. Similarly as in Example 2.5, we get the necessary condition for a local minimiser in this case:

$$
\max _{\alpha \in Q_{0}} f_{\alpha}^{(k)}\left(x_{0}\right) d^{k} \geqslant 0
$$

for every $d \in K_{C}\left(x_{0}\right)$ with

$$
\max _{\alpha \in Q_{0}} f_{\alpha}^{(i)}\left(x_{0}\right) d^{i} \leqslant 0 \quad \forall i \in I \backslash\{k\}, \quad \max _{\beta \in B_{0}} g_{\beta}^{(j)}\left(x_{0}\right) d^{j} \leqslant 0 \quad \forall j \in J .
$$

\section{Higher-Order Sufficient Optimality Conditions}

In this section we assume that $X=\mathbb{R}^{m}$.

DEFINITION 3.1: [10] The point $x_{0} \in D$ is said to be a strict local minimiser of order $k$ for the mathematical program $\min \{F(x) \mid x \in D\}$ if there exist $\sigma>0$ and a neighbourhood $U$ of $x_{0}$ such that

$$
F(x) \geqslant F\left(x_{0}\right)+\sigma\left\|x-x_{0}\right\|^{k} \quad \forall x \in U \cap D .
$$

Let $x_{0}$ be a feasible point for (P). As in the previous section, we consider realvalued functions $\varphi_{\alpha}^{(i)}, \alpha \in Q, i \in I$, and $\psi_{\beta}^{(j)}, \beta \in B, j \in J$, on $\mathbb{R}^{m}$, and we define

$$
M\left(x_{0}\right):=\left\{d \in K_{C}\left(x_{0}\right) \mid \sup _{\alpha \in Q_{0}} \varphi_{\alpha}^{(i)}(d) \leqslant 0 \forall i \in I \backslash\{k\}, \sup _{\beta \in B_{0}} \psi_{\beta}^{(j)}(d) \leqslant 0 \forall j \in J\right\} .
$$

Let us introduce relations between $f_{\alpha}$ and $\varphi_{\alpha}^{(i)}, g_{\beta}$ and $\psi_{\beta}^{(j)}$.

BAsic Assumption 3.2. For all $d \in K_{C}\left(x_{0}\right)$ and sequences $d_{n} \rightarrow d, t_{n} \downarrow 0$ satisfying $x_{0}+t_{n} d_{n} \in C$ we have

$$
\begin{array}{ll}
\varphi_{\alpha}^{(i)}(d) \leqslant \limsup _{n \rightarrow \infty} \frac{1}{t_{n}^{i}}\left[f_{\alpha}\left(x_{0}+t_{n} d_{n}\right)-f_{\alpha}\left(x_{0}\right)\right] & \forall \alpha \in Q, i \in I, \\
\psi_{\beta}^{(j)}(d) \leqslant \limsup _{n \rightarrow \infty} \frac{1}{t_{n}^{j}}\left[g_{\beta}\left(x_{0}+t_{n} d_{n}\right)-g_{\beta}\left(x_{0}\right)\right] & \forall \beta \in B, j \in J .
\end{array}
$$

A higher-order sufficient optimality condition for (P) can be stated as follows. 
ThEOREM 3.3. Let $G\left(x_{0}\right)=0$ and let the basic assumption 3.2 be satisfied. Assume that

$$
\sup _{\alpha \in Q_{0}} \varphi_{\alpha}^{(k)}(d)>0 \quad \forall d \in M\left(x_{0}\right) \backslash\{0\}
$$

Then $x_{0}$ is a strict local minimiser of order $k$ for $(\mathrm{P})$.

Proof: Assume that $x_{0}$ is not a strict local minimiser of order $k$ for $(\mathrm{P})$. Then there exists a sequence $\left\{x_{n}\right\} \subseteq C \backslash\left\{x_{0}\right\}$ such that $G\left(x_{n}\right) \leqslant 0,\left\|x_{n}-x_{0}\right\| \leqslant 1 / n$, and $F\left(x_{n}\right)<F\left(x_{0}\right)+\left\|x_{n}-x_{0}\right\|^{k} / n$ for every $n$. Since $f_{\alpha}\left(x_{0}\right)=F\left(x_{0}\right)$ for $\alpha \in Q_{0}$, we obtain

$$
f_{\alpha}\left(x_{n}\right) \leqslant f_{\alpha}\left(x_{0}\right)+\left\|x_{n}-x_{0}\right\|^{k} / n \quad \forall \alpha \in Q_{0}, n \in \mathbb{N} .
$$

Let $t_{n}:=\left\|x_{n}-x_{0}\right\|$ and $d_{n}:=\left(x_{n}-x_{0}\right) / t_{n}$. Then $\left\|d_{n}\right\|=1$, so by the compactness of the unit sphere in $\mathbb{R}^{m}$ there exists a subsequence $\left\{d_{n_{\nu}}\right\}$ converging to $d$ with $\|d\|=1$. Since $t_{n_{\nu}} \downarrow 0$ and $x_{0}+t_{n} d_{n}=x_{n} \in C$, it follows that $d \in K_{C}\left(x_{0}\right)$.

Using $G\left(x_{0}\right)=0$ we have $g_{\beta}\left(x_{n}\right)-g_{\beta}\left(x_{0}\right)=g_{\beta}\left(x_{n}\right) \leqslant G\left(x_{n}\right) \leqslant 0$ for all $\beta \in B_{0}$, $n \in \mathbb{N}$. Thus

$$
\psi_{\beta}^{(j)}(d) \leqslant \limsup _{\nu \rightarrow \infty} \frac{g_{\beta}\left(x_{n_{\nu}}\right)-g_{\beta}\left(x_{0}\right)}{t_{n_{\nu}}^{k}} \leqslant 0 \quad \forall \beta \in B_{0}, j \in J
$$

by 3.2 . By combining (4) and 3.2 we obtain

$$
\begin{aligned}
\varphi_{\alpha}^{(i)}(d) & \leqslant \underset{\nu \rightarrow \infty}{\limsup } \frac{f_{\alpha}\left(x_{n_{\nu}}\right)-f_{\alpha}\left(x_{0}\right)}{t_{n_{\nu}}^{i}} \\
& \leqslant \limsup _{\nu \rightarrow \infty} \frac{\left\|x_{n_{\nu}}-x_{0}\right\|^{k}}{n_{\nu} t_{n_{\nu}}^{i}}=\lim _{\nu \rightarrow \infty} \frac{t_{n_{\nu}}^{k-i}}{n_{\nu}}=0 \quad \forall \alpha \in Q_{0}, i \in I .
\end{aligned}
$$

Hence $d \in M\left(x_{0}\right) \backslash\{0\}$ and $\sup _{\alpha \in Q_{0}} \varphi_{\alpha}^{(k)}(d) \leqslant 0$, which contradicts (3).

Theorem 3.3 includes [8] Corollary 2.1 as a special case.

\section{REFERENCES}

[1] A. Auslender, 'Stability in mathematical programming with nondifferentiable data', SIAM J. Control Optim. 22 (1984), 239-254.

[2] F.H. Clarke, Optimization and Nonsmooth Analysis (Wiley, New York, 1983).

[3] G. Heinecke and W. Oettli, 'Characterization of weakly efficient points', Z. Oper. Res. 32 (1988), 375-393. 
[4] A.D. Ioffe, 'Calculus of Dini subdifferentials of functions and contingent coderivatives of set-valued maps', Nonlinear Anal. 8 (1984), 517-539.

[5] A.D. Ioffe, 'Approximate subdifferentials and applications I: The finite dimensional theory', Trans. Amer. Math. Soc. 281 (1984), 389-416.

[6] D.V. Luu and W. Oettli, 'Necessary optimality conditions for nonsmooth minimax problems', Zeitschrift für Analysis und ihre Anwendungen 12 (1993), 709-721.

[7] L. Schwartz, Analyse mathématique I (Hermann, Paris, 1967).

[8] M. Studniarski, 'Necessary and sufficient conditions for isolated local minima of nonsmooth functions', SIAM J. Control Optim. 24 (1986), 1044-1049.

[9] D.E. Ward, 'Directional derivative calculus and optimality conditions in nonsmooth mathematical programming', J. Inform. Optim. Sci. 10 (1989), 81-96.

[10] D.E. Ward, 'Exact penalties and sufficient conditions for optimality in nonsmooth optimization', J. Optim. Theory Appl. 57 (1988), 485-499.

Institute of Mathematics

PO Box 631 BoHo

10000 Hanoi

Vietnam
Fakultät für Mathematik und Informatik

Universität Mannheim

68131 Mannheim

Germany

e-mail: oettli@math.uni-mannheim.de 